\title{
Germline variants in UNC13D and AP3B1 are enriched in COVID-19 patients experiencing severe cytokine storms
}

\author{
Hui Luo ${ }^{1}$ - Dan Liu ${ }^{2,3} \cdot$ Wenbing Liu',3,4 Gaoxiang Wang ${ }^{1} \cdot$ Liting Chen $^{1} \cdot$ Yang Cao $^{1} \cdot$ Jia Wei $^{1} \cdot$ Min Xiao $^{1} \cdot$ \\ Xin Liu $^{2,3,4} \cdot$ Gang Huang ${ }^{5}$. Wei Wang ${ }^{6}$ Jianfeng Zhou $\mathbb{1}^{1} \cdot$ Qian-fei Wang ${ }^{2,3,4}$
}

Received: 2 October 2020 / Revised: 16 March 2021 / Accepted: 1 April 2021 / Published online: 19 April 2021

(c) The Author(s) 2021. This article is published with open access

\begin{abstract}
Critically ill coronavirus disease 2019 (COVID-19) is characterized by severe cytokine storms, a hyperinflammatory condition intimately related to the development of fatal outcomes. Why some individuals seem particularly vulnerable to severe cytokine storms is still unknown. Primary immunodeficiency (PID)-related genes are inherited factors that dysregulate host inflammatory responses to infection, especially hemophagocytic lymphohistiocytosis (HLH)-related genes, established as contributors to the development of excessive cytokine storms. We analyzed the association between PID gene variants with severe cytokine storms in COVID-19. We conducted whole-exome sequencing in 233 hospitalized COVID-19 patients and identified four PID gene (UNC13D, AP3B1, RNF168, DHX58) variants were significantly enriched in COVID19 patients experiencing severe cytokine storms. The total percentage of COVID-19 patients with variants in UNC13D or $A P 3 B 1$, two typical HLH genes, was dramatically higher in high-level cytokine group than in low-level group (33.3 vs. $5.7 \%, P<0.001)$. Germline variants in $U N C 13 D$ and $A P 3 B 1$ were associated with the development of severe cytokine storms, fatal outcomes in COVID-19. These findings advance the understanding of individual susceptibility to severe cytokine storms and help optimize the current management of COVID-19.
\end{abstract}

\section{Introduction}

Coronavirus disease 2019 (COVID-19) is triggered by severe acute respiratory coronavirus 2 (SARS-CoV-2), taking on various degrees of severity, ranging from mild to

These authors contributed equally: Hui Luo, Dan Liu, Wenbing Liu

Supplementary information The online version contains supplementary material available at https://doi.org/10.1038/s41431021-00886-x.

Wei Wang
wwang@ vip.126.com
Jianfeng Zhou
jfzhou@tjh.tjmu.edu.cn
Qian-fei Wang
wangqf@ big.ac.cn
Department of Hematology, Tongji Hospital, Tongji Medical
College, Huazhong University of Science and Technology,
Wuhan, Hubei, China
CAS Key Laboratory of Genomic and Precision Medicine, Beijing critical illness [1]. Critically ill or intensive care unit (ICU)admitted COVID-19 is characterized by severe cytokine storms, a hyperinflammatory condition intimately related to the development of lethal acute respiratory distress syndrome, multiple organ failure, and even death [2, 3]. One question about the cytokine storms still lingers: why some individuals seem particularly vulnerable? Few relevant research studies are now underway exploring the underlying genetic mechanisms.

Germline variants of primary immunodeficiency (PID)related genes, including defects in innate and adaptive

Institute of Genomics, Chinese Academy of Sciences, Beijing, China

3 China National Center for Bioinformation, Beijing, China

4 University of Chinese Academy of Sciences, Beijing, China

5 Divisions of Pathology and Experimental Hematology and Cancer Biology, Cincinnati Children's Hospital Medical Center, Cincinnati, OH, USA

6 Department of Neurology, Tongji Hospital, Tongji Medical College, Huazhong University of Science and Technology, Wuhan, Hubei, China 
immunity, have been seen as major inherited factors that dysregulate host inflammatory responses to infection [4-6]. A set of 12 PID genes (PRF1, UNC13D, STX11, STXBP2, RAB27A, LYST, AP3B1, SH2D1A, BIRC4, ITK, CD27, and $M A G T 1)$ have been established as contributors to the development of cytokine storms, and are dubbed hemophagocytic lymphohistiocytosis (HLH)-related genes [7]. Despite their obvious clinical significance, the mutational status of PID genes, especially HLH genes has not been investigated in patients with COVID-19.

\section{Materials and methods}

\section{Patients and sample collection}

The study population consisted of 233 participants with newly diagnosed COVID-19 between January 13, 2020 and March 5, 2020 at Tongji Hospital, Huazhong University of Science \& Technology, Wuhan, China. Informed consent was obtained from each patient or patients' relatives. The detailed information was shown in Supplementary materials.

\section{High- and low-level cytokine groups}

The levels of serum cytokines were determined by Bio-Plex Pro Human Cytokines 48-Plex Screening (Bio-Rad Life Sciences, Hercules, CA, USA) on a Luminex FIEXMAP 3D system (Luminex, Austin, TX, USA) by following the manufacturer's protocols. The relative levels (fold change relative to healthy donors) of IP-10, IL-Ra, or MCP-3 were used to perform receiver operating characteristic (ROC) curve analysis to discriminate between ICU and non-ICU patients. ICU admission, as a status variable, was used in ROC analysis since COVID-19 patients admitted to ICU tended to experience severe cytokine storms.

\section{Exome sequencing and rare, damaging variants filtering}

All coding variants were generated from whole-exome sequencing (WES) or targeted exome sequencing of DNA samples. We constructed a knowledge base of 241 genes involved in host antiviral immunity (Table S1). The rare variants (minor allele frequency $(\mathrm{MAF}) \leq 0.01$ in overall populations from public database $1000 \mathrm{G}$ and gnomAD) were identified. The damaging variants meant having a functional impact of the proteins and were predicted by in silico analysis based on PolyPhen-2, SIFT, and CADD. Variants were classified as damaging by at least two software predicted as damaging. The analysis strategies in detail were shown in Supplementary materials.

\section{Sequencing data availability}

The raw sequence data have been deposited in the Genome Sequence Archive [8] in NGDC [9], under accession number HRA000392. The variants data have been deposited in the LOVD database (https://databases.lovd. nl/) and GVM database [10] (https://bigd.big.ac.cn/gvm/ getProjectDetail?project=GVM000127).

\section{Results}

We performed WES on 233 hospitalized COVID-19 patients (mild, $n=58$; severe, $n=133$; critical, $n=42$ ) with an attempt to understand the correlation between PID genes and severe cytokine storms in COVID-19. The median age was 60 years (interquartile range: 46-68 years). More clinical data are detailed in Table S2 and analysis strategies presented in Fig. S1.

The severity of cytokine storms in COVID-19 was rated in terms of three cytokines (IP-10, IL-1Ra, and MCP-3) as previously reported [11]. Patients were included in high-level cytokine group $(n=15)$ when all three cytokines were significantly increased (above the cutoff values), and the others were assigned to low-level cytokine group $(n=174)$, see Fig. 1A and Supplementary materials. There were no significant differences in the term of age, sex, comorbidity, duration of hospitalization between the high- and low-level cytokine groups, suggesting similar clinical baseline characteristics between the two groups (Table S3). We focused on PID genes by constructing a knowledge base of 241 antiviral immunity-related genes, which were mainly reported in PID diseases (see Supplementary materials and Table S1) [4]. We identified a total of 534 rare (MAF $\leq 0.01$ ), damaging variants in 145 PID genes from 233 COVID-19 patients (listed in Table S4). To find out genes most relevant to the severe cytokine storms in COVID-19 patients, the variant burden of each identified genes was compared between high- and lowlevel cytokine groups. Variants in four genes were found to be significantly enriched in high-level cytokine group. UNC13D (26.7\%, odds ratio $(\mathrm{OR})=6.7,95 \%$ confidence interval $(\mathrm{CI})=1.8-25.1)$ was the most frequently mutated gene, followed by AP3B1 $(13.3 \%, \mathrm{OR}=13.2,95 \% \mathrm{CI}=1.7-101.7)$, RNF168 (13.3\%, OR $=26.6,95 \% \mathrm{CI}=2.3-313.3)$, and DHX58 $(20.0 \%, \mathrm{OR}=6.0,95 \% \mathrm{CI}=1.4-26.0)$. The variant burdens of these four genes were also strikingly higher in highlevel cytokine group than in Chinese general population $(n=$ 301) from the 1000 Genomes project (Fig. 1B and Table S5). UNC13D (encoding Munc13-4) and AP3B1 (encoding AP3), two typical HLH genes, were among the major players in the development of HLH due to defects in T/NK-cell cytotoxic granule release pathway. Three heterozygous missense variants (NM_199242.3:c.1607G > T:p.(Arg536Leu) [12]， NM_1992 
A

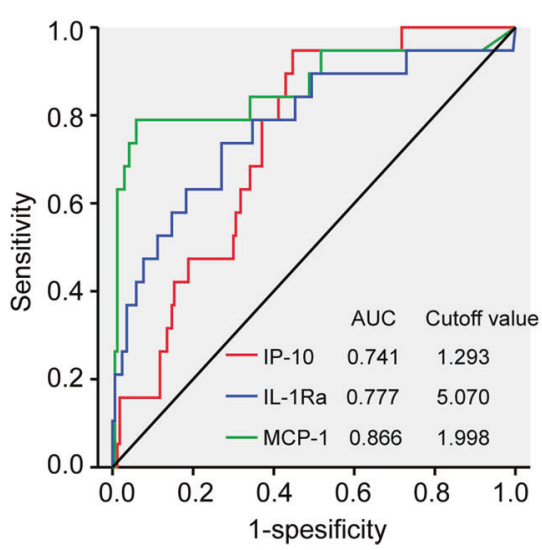

C

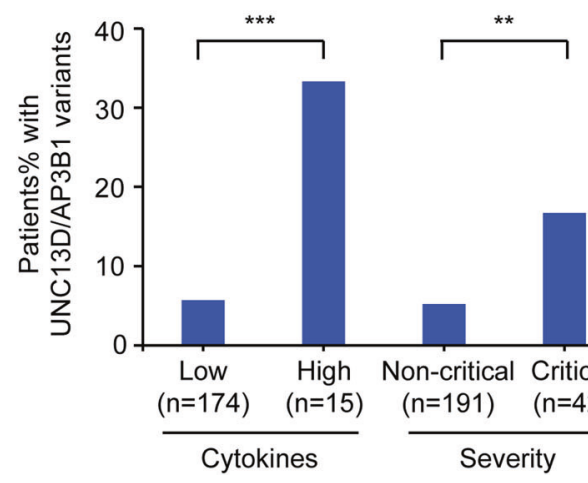

B
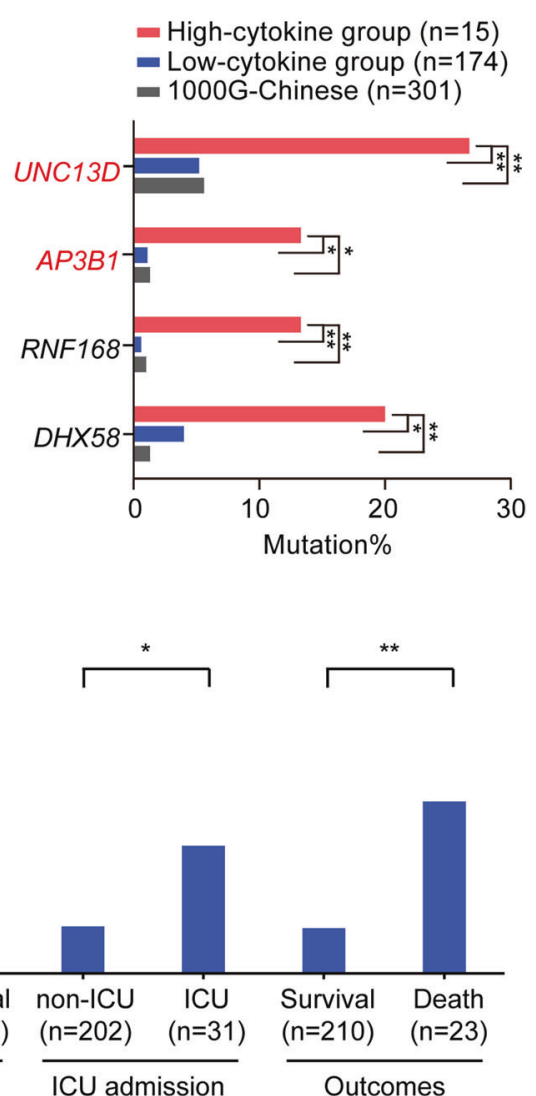

Fig. 1 Germline variants were significantly enriched in COVID-19 patients with severe cytokine storms. A The red, blue, and green lines represent the receiver operating characteristic (ROC) curves of IP-10, IL-Ra, or MCP-3 to discriminate COVID-19 patients with ICU admission or without ICU admission, respectively. The areas under the curve (AUC) and the optimal cutoff values set by Youden's index are shown in the graph. B The bar chart shows the mutation percentage of the four genes in high- and low-level cytokine groups and in Chinese

42.3:c.2588G > A:p.(Gly863Asp) [12, 13], and NM_199242.3: exon23:c.2242G > T:p.(Ala748Ser)) and one splice-site variant (NM_199242.3:exon17:c.1447-1G > A) in UNC13D and two heterozygous missense variants (NM_003664.5:exon23: c.2779G > A:p.(Gly927Ser), NM_003664.5:exon8:c.911 C > T:p.(Thr304lle)) in AP3B1 were found in high-level cytokine group. Among them, the NM_199242.3:c.2588G > A:p. (Gly863Asp) variant in $U N C 13 D$ is predictably affected the MHD2 domain by the HOPE software. In high-level cytokine group, one patient had two variants in the UNC13D gene, and four patients had a monoallelic variant in the $U N C 13 D$ or AP3B1 gene. The total percentage of COVID-19 patients with variants in UNC13D or AP3B1 was dramatically higher in high-level cytokine group than in low-level group (33.3 vs. $5.7 \%, P<0.001)$, in critical group than in non-critical group (16.7 vs. $5.2 \%, P<0.01)$, in ICU than in non-ICU admission group (16.1 vs. $5.9 \%, P<0.05$ ), and in death group than in survival group $(21.7$ vs. $5.7 \%, P<0.01)$ (Fig. 1C). To further validate our findings, we examined the variants in UNC13D population from $1000 \mathrm{G}$. The statistically significant mutation burden was analyzed by the chi-square test and the Fisher's test. $* P<0.05$; $* * P<0.01$. C The stacked bar shows the total percentage of patients harboring UNC13D or AP3B1 variants. The patients were grouped according to the severity of cytokine storms, COVID-19 severity, ICU admission, and fatal outcomes. The statistical significance between the groups was assessed by the chi-square test. ${ }^{*} P<0.05 ; * * P<0.01$; $* * * P<0.001$.

and AP3B1 genes by targeted sequencing in an additional outpatient group including 51 asymptomatic SARS-CoV-2 infection individuals. Only $2.0 \%$ (1 out of 51 ) of the individuals had the UNC13D variant, $3.9 \%$ (2 out of 51) had AP3B1 variants (Table S6). The total percentage of patients with UNC13D and AP3B1 variant in the outpatient group was much lower than in high-level cytokine group (5.9 vs. 33.3\%, $P<0.05$ ) (Fig. S3).

\section{Discussion}

Our study found that three types of PID gene variants were enriched in COVID-19 patients experiencing severe cytokine storms. UNC13D and AP3B1 variants might be associated with the development of severe cytokine storms, critical illness, and fatal outcomes in COVID-19 patients, while the functional relevance of two other type genes (antibody production gene RNF168 and viral sensor 
regulatory gene DHX58) warrants further investigation. A growing number of studies reported that heterozygous variants in HLH-related genes might be associated with HLH development [14-16]. Our study suggests that carrying a putatively deleterious variant in the UNC13D or AP3B1 gene might increase the risk of disease severity, possibly via interaction with other genetic mutations and/or nongenetic factors (e.g., exposure to SARS-CoV-2). Regarding the limitation of this study, we cannot differentiate functionally deleterious or neutral variants based on computational analysis. Except for the splicing site variant, all the other variants, including the in-frame variants with high CADD scores might be neutral when experimentally tested $[17,18]$ and the burden test should be further performed on experimentally proven deleterious variants. Our findings will be helpful in advancing the understanding of individual susceptibility to severe cytokine storms and optimizing the current management of COVID-19. For vulnerable populations genetically subject to the severe cytokine storms, more aggressive immunomodulation therapies should be considered [19].

Acknowledgements This work was supported by Emergency Research Project of Tongji Hospital of Huazhong University of Science and Technology (2020kfyXGYJ045 to JZ), Emergency Research Project of Hubei province (2020FCA006 to WW), Supported by Beijing Natural Science Foundation (M21022 to Q-fW), Key Program of the National Natural Science Foundation of China (81830008 to JZ), the National Natural Science Foundation of China (81873454 to XL), the Social Development, Project of Jiangsu Province (BE2017659 to Q-fW), the National Natural Science Foundation of China (81770211 to MX).

\section{Compliance with ethical standards}

Conflict of interest The authors declare no competing interests.

Ethical approval This study was approved by the Medical Ethics Committee of Tongji Hospital, Tongji Medical College, Huazhong University of Science and Technology, Wuhan, China (TJC20200113).

Publisher's note Springer Nature remains neutral with regard to jurisdictional claims in published maps and institutional affiliations.

Open Access This article is licensed under a Creative Commons Attribution 4.0 International License, which permits use, sharing, adaptation, distribution and reproduction in any medium or format, as long as you give appropriate credit to the original author(s) and the source, provide a link to the Creative Commons license, and indicate if changes were made. The images or other third party material in this article are included in the article's Creative Commons license, unless indicated otherwise in a credit line to the material. If material is not included in the article's Creative Commons license and your intended use is not permitted by statutory regulation or exceeds the permitted use, you will need to obtain permission directly from the copyright holder. To view a copy of this license, visit http://creativecommons. org/licenses/by/4.0/.

\section{References}

1. Guan WJ, Ni ZY, Hu Y, Liang WH, Ou CQ, He JX, et al. Clinical characteristics of coronavirus disease 2019 in China. N Engl J Med. 2020;382:1708-20.

2. Mehta P, McAuley DF, Brown M, Sanchez E, Tattersall RS, Manson JJ. COVID-19: consider cytokine storm syndromes and immunosuppression. Lancet. 2020;395:1033-4.

3. Moore JB, June $\mathrm{CH}$. Cytokine release syndrome in severe COVID-19. Science. 2020;368:473-4.

4. Bousfiha A, Jeddane L, Picard C, Ailal F, Bobby Gaspar H, AlHerz W, et al. The 2017 IUIS phenotypic classification for primary immunodeficiencies. J Clin Immunol. 2018;38:129-43.

5. Bousfiha A, Jeddane L, Picard C, Al-Herz W, Ailal F, Chatila T, et al. Human inborn errors of immunity: 2019 update of the IUIS phenotypical classification. J Clin Immunol. 2020;40:66-81.

6. Cunningham L, Simmonds P, Kimber I, Basketter DA, McFadden JP. Perforin and resistance to SARS coronavirus 2. J Allergy Clin Immunol. 2020;146:52-3.

7. Al-Samkari H, Berliner N. Hemophagocytic lymphohistiocytosis. Annu Rev Pathol. 2018;13:27-49.

8. Wang Y, Song F, Zhu J, Zhang S, Yang Y, Chen T, et al. GSA: genome sequence archive. Genom Proteom Bioinforma 2017;15:14-8.

9. CNCB-NGDC Members and Partners. Database Resources of the National Genomics Data Center, China National Center for Bioinformation in 2021. Nucleic Acids Res. 2021;49:D18-28.

10. Song S, Tian D, Li C, Tang B, Dong L, Xiao J, et al. Genome Variation Map: a data repository of genome variations in BIG Data Center. Nucleic Acids Res. 2018;46:D944-9.

11. Yang Y, Shen C, Li J, Yuan J, Wei J, Huang F, et al. Plasma IP-10 and MCP-3 levels are highly associated with disease severity and predict the progression of COVID-19. J Allergy Clin Immunol. 2020;146:119-27.e4.

12. Chen X, Wang F, Zhang Y, Teng W, Wang M, Nie D, et al. Genetic variant spectrum in 265 Chinese patients with hemophagocytic lymphohistiocytosis: molecular analyses of PRF1, UNC13D, STX11, STXBP2, SH2D1A, and XIAP. Clin Genet. 2018;94:200-12.

13. Miao Y, Zhu HY, Qiao C, Xia Y, Kong Y, Zou YX, et al. Pathogenic gene mutations or variants identified by targeted gene sequencing in adults with hemophagocytic lymphohistiocytosis. Front Immunol. 2019;10:395.

14. Zhang M, Bracaglia C, Prencipe G, Bemrich-Stolz CJ, Beukelman T, Dimmitt RA, et al. A heterozygous RAB27A mutation associated with delayed cytolytic granule polarization and hemophagocytic lymphohistiocytosis. J Immunol. 2016;196:2492-503.

15. Zhang K, Jordan MB, Marsh RA, Johnson JA, Kissell D, Meller J, et al. Hypomorphic mutations in PRF1, MUNC13-4, and STXBP2 are associated with adult-onset familial HLH. Blood. 2011;118:5794-8.

16. Cetica V, Sieni E, Pende D, Danesino C, De Fusco C, Locatelli F, et al. Genetic predisposition to hemophagocytic lymphohistiocytosis: report on 500 patients from the Italian registry. J Allergy Clin Immunol. 2016;137:188-96.

17. Yang R, Mele F, Worley L, Langlais D, Rosain J, Benhsaien I, et al. Human T-bet governs innate and innate-like adaptive IFN- $\gamma$ immunity against mycobacteria. Cell. 2020;183:1826-47.

18. Lamborn IT, Jing H, Zhang Y, Drutman SB, Abbott JK, Munir S, et al. Recurrent rhinovirus infections in a child with inherited MDA5 deficiency. J Exp Med. 2017;214:1949-72.

19. Ingraham NE, Lotfi-Emran S, Thielen BK, Techar K, Morris RS, Holtan SG, et al. Immunomodulation in COVID-19. Lancet Respir Med. 2020;8:544-6. 